\title{
World Society for Pediatric Infectious Diseases declaration on combating antimicrobial resistance in children
}

\author{
Jim Buttery ${ }^{1,2} \cdot$ Yonghong Yang ${ }^{3,4} \cdot$ Mike Sharland $^{5}$ on behalf of the World Society for Pediatric Infectious Diseases
}

Received: 18 September 2018 / Accepted: 21 September 2018 / Published online: 5 October 2018

(c) Children's Hospital, Zhejiang University School of Medicine 2018

\section{Preface}

The rise of antimicrobial resistance (AMR) is recognised as a major threat to global health. The United Nations' Sustainable Development Goals (SDG) do not explicitly address AMR, but many of the targets within the goals depend on effective therapy for infectious diseases. Notably, these include targets 3.1, 3.2, 3.3, and 3.8, relating to the reduction of early life mortality, eradicating ongoing epidemics including AIDS, tuberculosis and malaria, and achieving universal health coverage, as well as targets relating to poverty, malnourishment, and education [1]. The World Health Organization (WHO) recognised the outstanding importance of AMR by passing a Global Action Plan in 2015 and declaring AMR a global priority [2]. However, the WHO Global Action Plan on AMR mentions children only twice and to date includes no specific objectives or action points focusing on child health.

\section{Declaration}

The risk of acquiring and dying from infectious diseases is highest for neonates and children. The presentation and management of infections also differ between children and

Jim Buttery

jim.buttery@monash.edu

1 Department of Infection and Immunity, Monash Children's Hospital, 246 Clayton Road, Clayton, Melbourne, VIC 3168, Australia

2 Monash University, Melbourne, VIC, Australia

3 Beijing Children's Hospital Affiliated to Capital Medical University, Beijing, People's Republic of China

4 Shenzhen Children's Hospital, Shenzhen, People's Republic of China

5 Pediatric Infectious Diseases Research Group, St George's, University of London, London, UK adults, so that programs aiming to control and reduce AMR in adults may not be equally effective in children.

World Society for Pediatric Infectious Diseases (WSPID) calls upon global and national leaders and decision makers to recognise the distinctive nature and key importance of the impact of AMR in neonates and children. We propose that immediate action is needed to prevent a deterioration in global child health that could reverse much of the progress made in recent decades.

WSPID recommends that WHO consider specific strategies to combat AMR in neonates and children and routinely include neonatal and child-specific surveillance in the Global Antimicrobial Resistance Surveillance System (GLASS), or to establish independent Surveillance System specially for neonatal and child.

WSPID pledges to take action on the following points which are in line with the objectives of the 2015 WHO Global Action Plan on Antimicrobial Resistance and urges others to follow.

\section{Objectives and action points}

1. Improve awareness and understanding of antimicrobial resistance through effective communication, education and training

WSPID will promote awareness of AMR in children through advocacy and through formal statements and contributions to media covering the topic.

To promote best practice and current evidence, member society ESPID will provide its Online antimicrobial Management Course for a nominal fee to healthcare professionals from across the world. WSPID hopes other member societies also can provide such Online course in the future.

All members and all professional bodies are urged to work towards including modules or elements on infection control and appropriate use of antimicrobials in pediatric 
specialty training and in training for other specialties that regularly treat children, including general practice.

2. Strengthen the knowledge and evidence base through surveillance and research

We urge WHO and all public health authorities to collect and present neonatal and child-specific figures in all AMR surveillance activities.

WSPID will include as a key priority for WSPID support education and research addressing AMR in children. We request its member societies and ask other funders of research to follow in this effort. Topics of specific relevance include:

- antimicrobial resistance patterns in neonates and children and the risk factors and mechanisms for their emergence in different levels: global, regional, national, and local;

- optimal dosing of antimicrobials in neonates and children to maximise clinical outcomes, while reducing toxicity and the selection of AMR;

- monitoring patterns of both community and hospital prescription of antimicrobials to children to identify areas for improvement in current practice;

- limiting prescription of the Essential Medicines List for Children "Watch and Reserve" group antimicrobials where possible and appropriate;

- determining the reservoirs and circulation of drug-resistant strains in children with the aim of identifying the most suitable approaches for infection prevention, including vaccination.

3. Reduce the incidence of infection through effective sanitation, hygiene, and infection prevention measures

WSPID calls upon its members and member societies to initiate and support programs to promote breastfeeding and uptake of childhood and maternal vaccinations as methods to prevent infections in neonates and children and to reduce the antimicrobial use.

We further encourage health care providers to dedicate funds for appropriate staffing of services caring for those children most vulnerable to infection, including neonates and children who are immunocompromised or in intensive care, to enable staff to comply with hygiene and infection prevention measures to reduce healthcare-associated infections (HAI).

4. Optimise the use of antimicrobial medicines in human and animal health
All WSPID members are encouraged to contribute to the development of evidence based management guidelines for infectious diseases. These guidelines should reflect epidemiology and resistance patterns in neonates and children, as well as responsible and appropriate antimicrobial use.

WSPID and its member societies will advocate and support the implementation of pediatric antimicrobial stewardship programs for all levels of care, and will work to ensure that these practices are appropriate to the local context.

5. Develop the economic case for sustainable investment that takes account of the needs of all countries, and increase the investment in new medicines (including vaccines), diagnostic tools, and other interventions

We urge WSPID members to actively engage with key opinion leaders and industry to enhance current efforts in the licensing of medicines for children and neonates, the development of pediatric formulations and in providing equitable access to essential medicines. WSPID could consider developing a global advocacy group to work with regulators and industry partners to ensure that Pediatric Investigation Plans and Pediatric Study Plans are actually conducted in a timely manner. A clear goal would be to obtain pediatric regulatory approval for novel antimicrobial agents within 4 years of the adult licence being obtained. At the same time, the evidence base for the use of older antimicrobials should be developed through strategic clinical trials to define the best available treatment and improve the management of these severe infections in neonates and children.

Author contributions The preliminary draft of this Declaration was written by Professor MS and revised by Professor JB, the President of WSPID, Professor YY and other board members of the society. It was approved by the Board Meeting of WSPID on December 5, 2017, in Shenzhen, China.

Funding None.

Ethical approval Not needed.

Conflict of interest The authors declare that they have no competing interests.

\section{References}

1. United Nations; 2015. https://www.un.org/sustainabledevelopme nt/health/. Accessed 15 Aug 2018.

2. World Health Organization. Global action plan on antimicrobial resistance. Geneva: World Health Organization; 2015. 